\title{
Soil microbial inoculation increases corn yield and insect attack
}

\author{
Lea Megali ${ }^{1} \cdot$ Benjamin Schlau $^{2} \cdot$ Sergio Rasmann ${ }^{2,3}$
}

Accepted: 11 June 2015 /Published online: 4 July 2015

(C) INRA and Springer-Verlag France 2015

\begin{abstract}
Effective Microorganisms $\left(\mathrm{EM}^{\circledR}\right)$ is a "biofertiliser" soil inoculant, marketed as a crop yield enhancer. However, the literature has neither comprehensively reviewed its purported effects on harvests across multiple species nor investigated its effects on plant herbivore defence other than this group's previous research on tomatoes. Here a meta-analysis of 39 journal articles and a greenhouse experiment with nine crop species afforded a nuanced assessment of Effective Microorganisms' effects on plant growth and yield. Overall, in line with predictions, Effective Microorganisms showed significant positive effect on yield and growth $(0.03$ effect sizes increase) in the meta-analysis, and increased growth $16 \%$ in the greenhouse, but with strong, and at times negative, species-specific responses. An additional potential benefit of Effective Microorganisms includes increased defence against herbivore attack, but inoculated corn (Zea mays) in a field and a greenhouse experiment exhibited decreased defences. Specifically, the field experiment demonstrated that Effective Microorganisms treatment corresponded to a $26 \%$ reduction in predatory insect diversity on corn plants, while not improving growth or yield but did increase water uptake. A subsequent greenhouse experiment suggested likely physiological mechanisms behind the loss of predator diversity. When non-inoculated control corn plants were set upon by
\end{abstract}

Sergio Rasmann

sergio.rasmann@unine.ch

1 Department of Ecology and Evolution, University of Lausanne UNIL Sorge, Le Biophore, CH-1015 Lausanne, Switzerland

2 Ecology and Evolutionary Biology, University of California, Irvine, CA, USA

3 Present address: Institute of Biology, University of Neuchâtel, CH-2000 Neuchâtel, Switzerland caterpillars of the herbivorous insect Spodoptera littoralis, the plants increased production of defensive volatile organic compounds (VOCs) by $272 \%$. Surprisingly, inoculation with Effective Microorganisms rendered greenhouse corn plants $51 \%$ more palatable to $S$. littoralis. Further localised studies are, therefore, needed to efficiently incorporate Effective Microorganisms with either conventional or sustainable agricultural management systems.

Keywords Insect herbivores $\cdot$ Indirect defences $\cdot$ Volatile organic compounds $\cdot$ Plant defences $\cdot$ Induced resistance . Plant-microbes interaction $\cdot$ Predators $\cdot$ Sustainable agriculture

\section{Introduction}

To meet the food demands of a world population likely to surpass 9.6 billion by 2100 , and answer a lesser but growing demand for biofuels, farmers worldwide have been intensifying agricultural output through ever-increasing application of chemical fertilisers and suites of synthetic pesticides. Advances in agricultural technologies have allowed the doubling of cereal output over the last four decades, and so far, have more than adequately met the consumption demands of those with access to food distribution networks and sufficient economic resources (Tilman et al. 2002). However, in addition to the retail costs of foodstuffs, intensification of agricultural systems exacts a less-obvious price through degradation of soil quality (Stoate et al. 2001), downstream eutrophication of waterways (Vitousek et al. 1997), loss of biodiversity (Green et al. 2005) and exposure of farmhands to carcinogenic pesticides (Gevao et al. 2000). One of the major challenges for a global society in the coming decades is to continue to exceed agricultural market pressures while reducing negative impacts 
on ecosystems and human health (Foley et al. 2011). To meet this challenge, a growing number of farmers are implementing "sustainable agriculture" techniques, characterised by employing biofertilisers and crop rotations, as well as reducing degradation of biodiversity, water quality and soil health (Altieri 1995; Kong 2014).

Ensuring fertile and resilient soil microbe habitat may be essential elements of insuring continuation of belowground ecosystem services that support agriculture. Complex interactions between soil-borne microbes (e.g. endophytic fungi, mycorrhizae, rhizobacteria, rhizobia) and plants increase plant vigour and survival through direct effects and indirect mechanisms. Directly, soil microbes can form symbiosis with the plant to increase nutrient acquisition and plant vigour (Compant et al. 2005; Pineda et al. 2010), while indirectly, soil microbes are essential to recirculating nutrients that would otherwise be locked up in undecomposed detritus (Adesemoye and Kloepper 2009). Additionally, a surge of recent studies is showing that beneficial microbial soil communities play roles in above- and belowground defences against herbivores (Pineda et al. 2010) as well as resistance to pathogens (van de Voorde et al. 2012).

In answer to a recent but growing demand among sustainable farmers into the use of soil inoculants for improvement of long-term soil productivity (Johansson et al. 2004; Bashan 1998), microbial biofertilisers have been marketed as ecologically friendly alternatives to conventional methods of nutrient and pest management (Javaid 2010). One such biofertiliser is Effective Microorganisms $\left(\mathrm{EM}^{\circledR}\right)$, developed in the 1990 s by Professor Teruo Higa at the University of the Ryukyus, Okinaw, Japan (Higa 2000). Effective Microorganisms inoculant is primarily constituted of lactic acid bacteria, yeasts and phototrophic bacteria (Higa 2000). Effective Microorganisms are commercially available worldwide and are of high potential for use in developing countries (Higa 2000). Research by Cóndor Golec et al. (2007) highlighted Effective Microorganisms' effects on soil quality, and, in some cases but not always, improved plant growth and crop yield (see, e.g. Mayer et al. 2010). In addition, these studies and others have not assessed Effective Microorganisms' potential for enhancing or reducing plant defence and resistance against herbivore pests. To our knowledge, only one recent paper has shown that Effective Microorganisms inoculation of tomato plants not only promotes increased yield but also increases susceptibility to Spodoptera littoralis caterpillars via inhibition of chemical defence production (Megali et al. 2014).

When herbivores attack plant tissue, plants can defend themselves with constitutive defences, or as an adaptive means of conserving resources, induce production of defensive secondary metabolites in a timely response to herbivory
(Karban and Baldwin 1997). For example, herbivore attack induces the upregulation of toxic chemicals that directly affect herbivore performance (i.e. direct defences). Indirect defences include release volatile organic compounds that are used by predators and parasitoids to locate their prey and hosts on the plant (Turlings and Wäckers 2004). Pineda et al. (2010) has highlighted the importance of microbial-mediated positive effects on induction of both direct and indirect plant defences. And while previous research found that Effective Microorganisms reduced defence and resistance in tomatoes (Megali et al. 2014), Effective Micoorganisms' effects on other traits (such as growth and yield) appear to be highly species-specific (see for instance the meta-analysis presented here). We therefore hypothesise this specificity may hold true for traits associated with plant defence as well.

The aims of this study were therefore to test whether Effective Microorganisms is able to (1) increase yields for multiple crop species, and (2) simultaneously improve plant resistance against herbivore attack in corn (Zea mays). The effect of Effective Microorganisms on plant growth and yield were reviewed via a meta-analysis of the literature. Next, the interactive effects of Effective Microorganisms and chemical fertiliser application on nine species of crops were assessed (Fig. 1). Finally, Effective Microorgnisms' effects on predator recruitment and herbivore resistance were investigated through a field manipulation and greenhouse experiment of corn plants. We expected that plants inoculated with Effective Microorganisms would perform as good as when inoculated with chemical fertilisers, and because of increased plant vigour, Effective Microorganisms-inoculated corn would be more resistant against insect herbivores than non-inoculated corn.

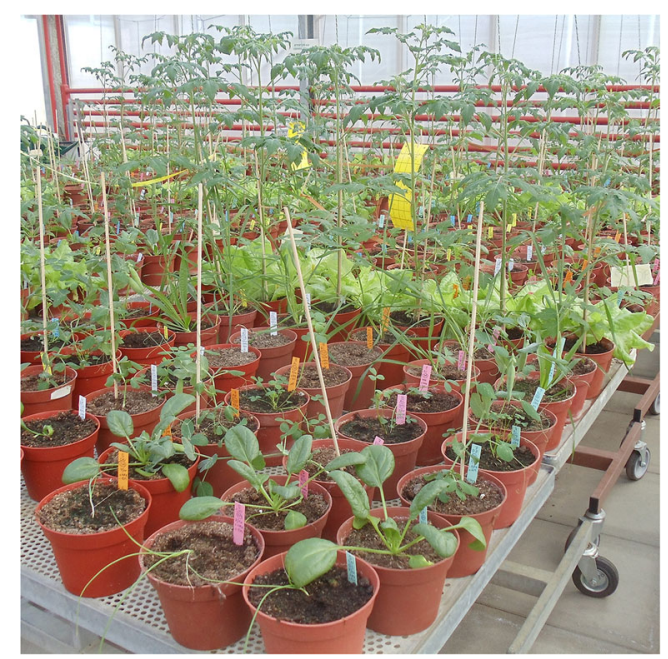

Fig. 1 Greenhouse experiment with nine crop species. Plants were placed side by side, applied with four treatments, including: control unfertilised plants, plants fertilised with Effective Microorganisms (EM), plants fertilised with NPK, and plants fertilised with both EM and NPK. Plants were randomly distributed on the benches within the greenhouse 


\section{Material and methods}

\subsection{Effective microorganisms}

Effective Microorganisms is primarily composed of lactic acid bacteria (viz., Lactobacillus plantarum, Lactobacillus casei and Streptococcus lactis), yeasts (Saccharomyces spp.), phototrophic bacteria (viz., Rhodopseudomonas plastris and Rhodobacter sphacrodes) and actinomycetes (Streptomyces spp.) (Cóndor Golec et al. 2007; Higa 2000). The microbial mixture is commercially available as a concentrated liquid suspension (EM-1), which is the base substrate for several additional commercially available byproducs such as EMA - with sugarcane molasses and water, EM-5 - with sugarcane molasses, water, ethanol and vinegar; or Bokashi (i.e. fermented organic matter, in this case with sugarcane molasses and a fermentable organic substrate). All our experiments were performed using Bokashi mixed with potting soil, and EM-1 as liquid soil inoculation (see below) provided by EM Schweiz AG, Switzerland.

\subsection{Effect of Effective Microorganisms on plant growth and yield—a meta-analysis}

To assess the effects of Effective Microorganisms on plant growth and yield across a wide range of studies, a comprehensive meta-analysis of the available literature was performed. All papers published on Effective Microorganisms were searched by means of Thompson Reuters search engine (Web of Science) using keywords "effective" and "microorganism" and "EM" in all combinations. The search was next expanded with the software Google Scholar and using keywords "EM," "Effective Microorganisms", "agriculture" and "yield". Only studies that measured yield (number and weight of seeds and fruits) and plant growth (plant height, plant biomass, root biomass) were included. To avoid confounding effects of other soil amendments, only studies that specifically tested Effective Microorganisms' effects against a control (untreated) treatment, but that maintained the same soil substrate, were included.

For each response variable, the average value for both treatments (with or without Effective Microorganisms) was extracted from tables or, when tables were missing, were digitalized from graphs using the graph digitizer software Grab it! (www.datatrendsoftware.com/home.html). The two response variables for individual plant species were retained, in which all measures of plant biomass (including roots and shoots), plant height, root length or numbers of leaves were classified as growth; and whereas all measures of fruit or grain number and weight were classified as yield.

Because most of the studies did not provide sample size, nor standard deviation, hypotheses were tested using an unweighted log-response-ratio (LLR), calculated from mean responses in the presence of Effective Microorganisms $(\mathrm{EM}+)$ and absence of Effective Microorganisms (EM-) on plants, and calculated as $L L R=\ln (E M+/ E M-)$ for each individual plant species.

One-way analyses of variance (ANOVAs) were used to test species' effect of Effective Microorganisms on growth and yield. Secondly, the effect of Effective Microorganisms on yield and growth on different plant species and on the total mean was considered positive, negative or neutral, based on deviation of means from the zero value using $t$ tests. All analyses were done in R (Team 2011).

\subsection{Effect of Effective Microorganisms on plant growth-a greenhouse experiment}

The greenhouse experiment was designed to test the interactive effect of Effective Microorganisms and conventional chemical fertiliser on plant growth. Nine different crop species were analysed: alfa-alfa (Medicago sativa), carrot (Daucus carota), corn (Z. mays), lettuce (Lactuca sativa), onion (Allium cepa), green peas (Pisum savitum), spinach (Spinacia oleracea), tomato (Solanum lycopersicum) and wheat (Triticum sativum). Each species was attributed to four treatments by manipulating Effective Microorganisms and chemical fertiliser (NPK hereafter) in a fully factorial design, including (1) control, untreated plants, (2) Bokashi (Effective Microorganisms), (3) NPK and (4) Bokashi+NPK. Seeds of ten plants per species and per treatment were germinated in autoclaved medium-low $P$ potting soil (Orbo-2, Schweizer AG, Lausanne, Switzerland) with perlite (3:1). After 10 days, plantlets were transplanted into $15-\mathrm{cm}$ diameter plastic pots containing the same soil used for germination and placed in a greenhouse at $25 / 18^{\circ} \mathrm{C}$ (day/night temperature), $60 \%$ relative humidity and a photoperiod consisting of $14 \mathrm{~h}$ of daylight. According to commercial directives, Effective Microorganisms treatment consisted of $20 \mathrm{~g}$ of the solid Bokashi commercial preparation (EM Schweiz AG, Bern, Switzerland) placed at the bottom of the pots prior to transplanting of germinated seedlings. In addition, $50 \mathrm{ml}$ of Bokashi liquid (1:1000) was applied once a week. Chemical fertilizer (NPK; $50 \mathrm{ml}$ of $1 \%$ preparation, Landi, Switzerland) was applied once a week. An additional $50 \mathrm{ml}$ of tap water was added to each pot during the treatment application to mimic the double treatment (Bokashi plus NPK). Control plants received only the equivalent amount of tap water. After a month of growth post-germination, plants were harvested, dried at $50{ }^{\circ} \mathrm{C}$ for 1 week and weighed.

The interactive effect of Effective Microorganisms, NPK and species identity on plant biomass was estimated with three-way permutation ANOVA using the oavp function in lmPerm package in $\mathrm{R}$ (Wheeler 2010). Permanova are used when data deviate from normality assumptions. Individual treatment effects were assessed with Tukey HSD post hoc tests for each species. 


\subsection{Effect of Effective Microorganisms application on insect communities in cornfields}

In order to study Effective Microorganisms' influence on insect communities around crop fields, a field experiment was established at the EM Schweiz production in Bern, Switzerland $\left(46^{\circ} 56.02 .9 \mathrm{~N}, 07^{\circ} 39.35 .4 \mathrm{E}\right)$. A normal rotational crop field ( $24 \mathrm{~m}$ long $\times 15 \mathrm{~m}$ wide) was sown with corn seeds (Z. mays, var. Fabregas) on 15 April 2012. The distance between the rows was $75 \mathrm{~cm}$, and the distance between plants was $10-13 \mathrm{~cm}$. The field was separated into 12 rectangles of $5 \times 6 \mathrm{~m}$. Each rectangle (hereafter referred to as plot) was isolated from another with a buffer zone of $2 \mathrm{~m}$. Two treatments in a checkerboard design were applied to the field (1) with Effective Microorganisms-Bokashi and (2) a control treatment without Effective Microorganisms. Before sowing, Effective Microorganisms-Bokashi treatment was applied a first time at a rate of $1 \mathrm{~kg}$ of solid Bokashi $/ \mathrm{m}^{2}$. After planting, soil around corn plants was sprayed twice (once when plants were $15-20 \mathrm{~cm}$ high, and once when they were $40-50 \mathrm{~cm}$ high) with the EM-A diluted 1:1 with water at a dosage of $20 \mathrm{l} / \mathrm{ha}$. For the control treatment, only water was applied. All the analyses were done on ten randomly chosen plants per square ( $n=60$ plants per treatment). After 70 days of growth, plant performance was assessed by measuring the chlorophyll content (three measures per leaf for each plant measured with a SPAD-502Plus chlorophyll-metre (Konica Minolta, China, Investment, Ltd)) and the height of the plants. After about 5 months of growth, the selected 120 plants were harvested. Each plant was cut at soil level, and all the ears of each plant were separated and dried at $30{ }^{\circ} \mathrm{C}$ for 2 weeks. Seeds were removed and weighed. The entire maize plant, without its ear was weighed a first time directly after the harvest and a second time 2 weeks later when the plants were totally dried to obtain water content per plant. Treatment differences for each response variable (chlorophyll level, height, seed mass, dry biomass and water content) were analysed with mixed-model analyses and included the following factors: treatment (with or without Effective Microorganisms) and plots nested within treatments (planting squares random effect). Data were logtransformed where needed to meet normality assumptions.

Subsequently, in order to observe whether the Effective Microorganisms application could influence insect abundance and diversity aboveground, yellow sticky insect traps were assigned to two randomly chosen plants at the centre of each square during 1 week in July. Trapped insects were identified and divided into orders and family whenever possible, and into three functional groups, including herbivores, predators, and all other insects not belonging to a classical herbivore or predator group.

To assess if Effective Microorganisms influenced the composition and (i.e. identity and relative abundance) of aboveground insects in cornfields, Mantel tests were performed with the vegdist function of the vegan package of $\mathrm{R}$ (Oksanen et al.
2013) on the distance matrices of insects found near plants belonging to the two treatments (Effective Microorganisms-Bokashi and control). The Bray-Curtis metric was used to calculate dissimilarity among samples. Next, we assessed the difference in total abundance and diversity, as measured using the Shannon diversity index, of insects between the two samples with a permutation ANOVA using the oavp function in lmPerm package of $\mathrm{R}$ (Wheeler 2010).

\subsection{Effects of Effective Microorganisms on corn resistance and defences}

Because Effective Microorganisms' treatment effect on the attraction of predators to plants in the field (see Sect. 3) was observed, the influence on volatile organic compound emissions from corn plants (i.e. indirect defences) as well as plant resistance to herbivores (i.e. direct defences) were investigated with a subsequent greenhouse experiment. Twenty plants of the same hybrids used for the field experiment were germinated and grown in greenhouses as described above for the nine species experiment. Half of the plants were inoculated with Effective Microorganisms. The other half was left un-inoculated (control). After 2 weeks of growth, half of the plants were inoculated with five first instar larvae of the Egyptian cotton leafworm S. littoralis (Lepidoptera: Noctuidae), a highly polyphagous nocturnal moth originating from Northern Africa and the Mediterranean Basin and a major pest of crop plants, including corn.

On the fourth day, every plant was completely covered with a nalophan bag and leaf volatiles were collected by pulling air through a charcoal filter trap (Orbo-32; Supelco, Bellefonte, PA, USA) with a flow rate of $200 \mathrm{ml} \mathrm{min}^{-1}$ for $4 \mathrm{~h}$. Traps were eluted with $350 \mu \mathrm{l}$ of dichloromethane after the addition of $5 \mu \mathrm{l}$ of tetraline $(50 \mathrm{ng} / \mu \mathrm{l})$ as an internal standard, and $1-\mu \mathrm{l}$ aliquots of each sample were analysed by gas chromatography mass spectrometry (GCMS ThermoGC coupled to a DSQ MS, Thermo Scientific) equipped with a DB-5 ms column (30 m, $0.25 \mathrm{~mm}$ internal diameter, $0.25 \mathrm{~lm}$ film thickness; Thermo). Helium was used as carrier gas at a constant flow of $1.2 \mathrm{ml} \mathrm{min}^{-1}$ and the following column temperature gradient, $40{ }^{\circ} \mathrm{C}$ for $5 \mathrm{~min}$, increased to $180{ }^{\circ} \mathrm{C}$ at $10^{\circ} \mathrm{C} \mathrm{min}^{-1}$, increased to $220{ }^{\circ} \mathrm{C}$ at $40-1{ }^{\circ} \mathrm{C} \min ^{-1}$, followed by a final hold at $220^{\circ} \mathrm{C}$ for $10 \mathrm{~min}$. Peak areas were integrated from selected ion chromatograms specific to the individual compounds and normalised by the peak area of the internal standard. Peaks were identified where possible by comparison of retention times and mass spectra with standards. Volatile emissions were reported as micrograms tetraline equivalents.

Differences in volatile organic compounds composition among herbivore treatment, Effective Microorganisms treatment, and their interaction were tested using a permutational ANOVA (PERMANOVA), using the Adonis function in the vegan package of R (Oksanen et al. 2013). The Bray-Curtis metric was used to calculate dissimilarity among samples. 
Larvae were subsequently left feeding for another 3 days before being collected, oven-dried and weighed as an assessment of plant resistance against chewing herbivores. After larvae removal, plant dry biomass was measured. The effects of Effective Microorganisms application on both larval and plant biomass were assessed with one-way ANOVAs.

\section{Results and discussion}

\subsection{Literature review}

The initial bibliographic database search yielded 72 articles, of which 39 were kept based on eligibility criteria. Across all studies 25 plant species have been used multiple times leading to a total of 914 datapoints. Maize (Z. mays), onion (A. cepa), rice (Oryza sativa) and mung bean (Vigna radiate) were the species most extensively studied. Across all species, Effective Micoorganisms application increased the growth response ratio by 0.02 effect sizes and yield of plants by 0.03 effect sizes (Fig. 2). Nevertheless, we found strong species-specific

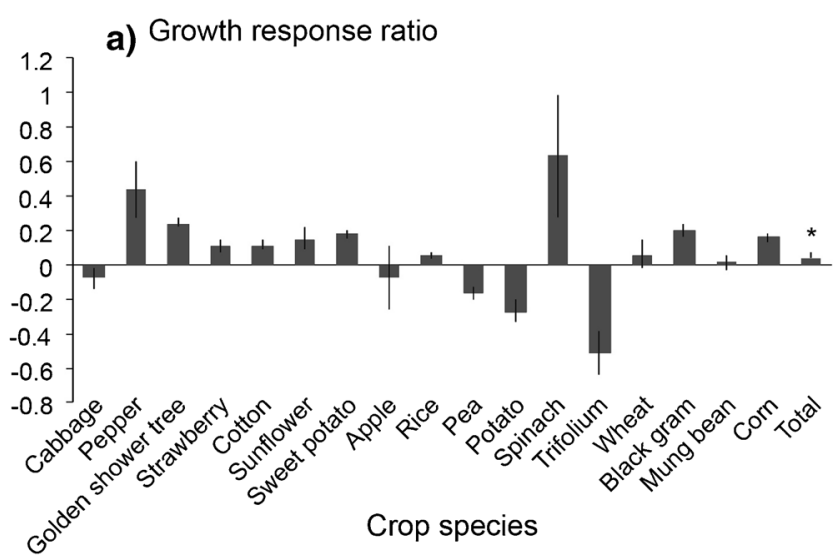

b) Yield response ratio

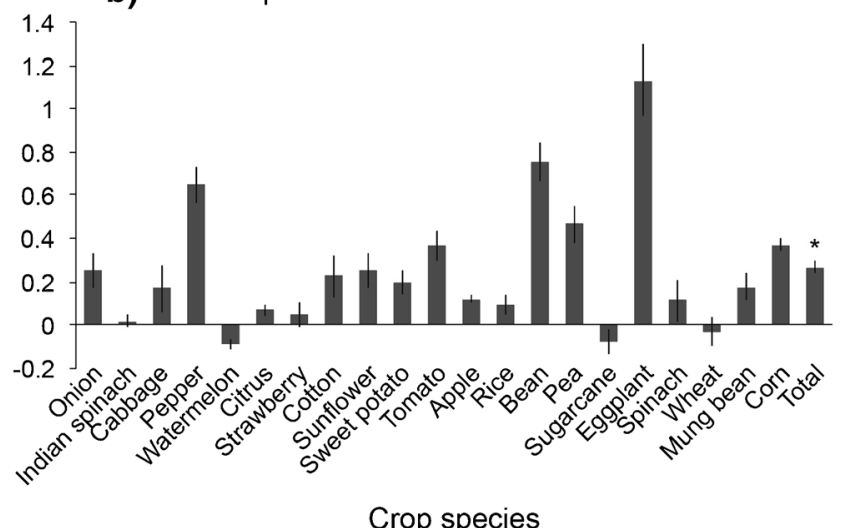

Fig. 2 Response ratio of crop species inoculated with Effective Microorganisms (EM) treatment relative to controls for a growth and $\mathbf{b}$ yield from meta-analysis. Bars are mean $\pm 1 \mathrm{SE}$, asterisks above error bars indicate significant EM treatment effect $(p<0.05$, single-sample $t$ test, data not shown) response variation to treatment both for growth (Fig. 2a, $F_{15}$, ${ }_{498}=7.55, p<0.0001$ ) and for yield (Fig. $2 b, F_{21}, 377=10.23$, $p<0.0001$ ).

Studies that met our criteria originated from soil ecosystems in tropical and temperate climates, which differ considerably in terms of moisture, $\mathrm{pH}$ and availability of organic nutrients. These biotic factors have been shown to strongly influence belowground microbial community composition (Lentendu et al. 2014) and may affect Effective Microorganisms' ability to establish or sufficiently improve inoculated soils. Despite this heterogeneity in soil environments, a strong majority of crops exhibited a significant increase for growth and yield when treated with Effective Microorganisms, suggesting that soil inoculations could be efficiently used on a wide variety of systems.

The fact that not all species responded positively to Effective Microorganisms application (see, e.g. the negative effect of Effective Microorganisms on watermelon, cabbage, pea and trifolium) suggests that (1) not all plant species are responsive to Effective Microorganisms effects on soil (Hayat et al. 2010) or (2) soil inoculation might not have resulted in the establishment of Effective Microorganism's constitutive species into the local soil microbe community (Mayer et al. 2010). In support of the latter possibility is the high variation across studies in the response variables measured within a species (e.g. the high variation in responses for worldwide planted crops such as wheat). Therefore, local soil ecologies may be an important factor to consider in future investigations into, or applications of, Effective Microorganisms as well as other beneficial microorganism biofertilisers. However, local soil ecology may be less of a determining factor compared with species-specific plant-Effective Microorganisms interactions. As is seen in our greenhouse experiment, not all tested crop species respond similarly to Effective Microorganisms treatment when abiotic factors are kept constant (see below). The greenhouse experiment results thus reinforce the importance of species-specific responses, whether positive, negative, or none at all, as stronger determining factors than local soil ecology in regard to crop performance.

\subsection{Greenhouse experiment}

Across the nine species tested, Effective Microorganisms inoculation significantly increased dry biomass of the plants by $16 \%$ (Fig. 3, EM effect; $p<0.0001$ ), whereas chemical fertiliser application had no overall effect on plant biomass (Fig. 3, NPK effect; $p=0.27$, NPK $\times$ EM effect; $p=0.36$ ). Species also responded differently to Effective Microorganisms' application (species effect; $p<0.0001, \mathrm{EM} \times$ species effect; $p<0.0001 ; \mathrm{NPK} \times$ species effect; $p=1$ and species $\times \mathrm{NPK} \times$ EM effect, $p=1$ ), with tomato, peas and spinach plants being the most responsive to the microbial inoculum (Fig. 3). 


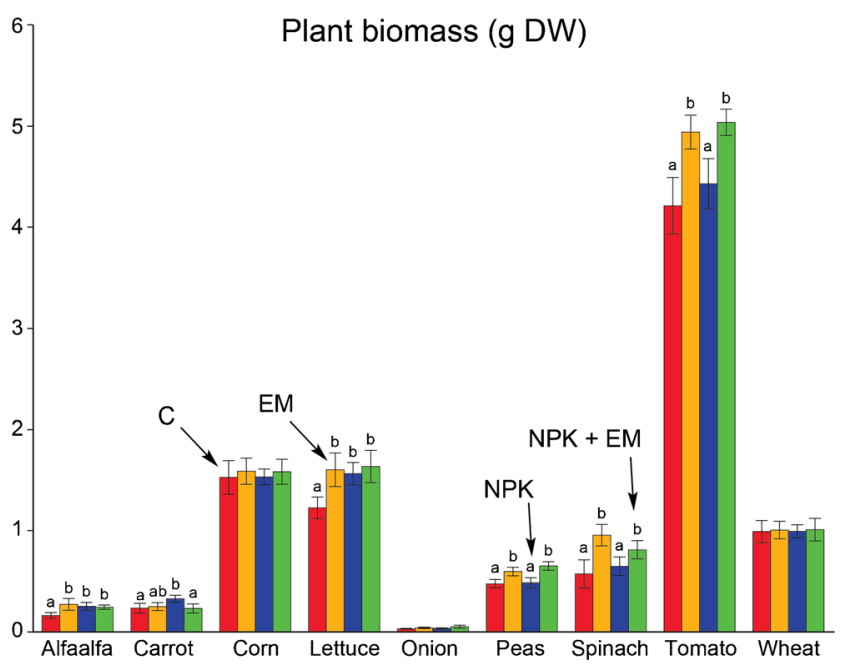

Fig. 3 Mean shoot weight of nine species of plants grown under different fertilisation treatments, including unfertilised control plants (red bars), plants inoculated with Effective Microorganisms (EM; orange bars), fertilised plants (blue bars), and plants inoculated with EM and fertilised (green bars). Bars are mean $\pm \mathrm{SE}$, letters above bars indicate significant treatment effect within individual species $(p<0.05$, Tukey HSD test)

These results, in addition to the literature review, are in accordance with the predicted effects of Effective Microorganisms on plant performance, and also suggest a species-specific response to Effective Microorganisms treatment. While the variety of crop plants investigated were all grown for 37 days and certain species may necessitate more growing time to exhibit symptoms of nutrient stress, a few species had adequate time for soil treatments to exhibit effects on plant performance. Spinach, tomatoes and peas, for example, displayed a significantly greater increase in biomass when under Effective Microorganisms treatment, even outperforming application of conventional fertiliser. Effective Microorganisms-treated alfalfa and lettuce performed similarly as when applied with conventional fertiliser, but better than when left untreated. Other species showed no difference in biomass in response to treatments. In regard to corn, the field experiment (see below) corresponded to the plant's performance in the greenhouse, with no increase in biomass, even after growing to harvest in the field. As mentioned above, Effective Microorganisms' effect could be dampened or modified depending on the initial soil substrate, which leaves open the possibility that further greenhouse testing with multiple soil profiles could indicate an interactive effect of Effective Microorganisms and soil type.

Others argue that the Effective Microorganisms' effects on plant performance are independent of the microbes, but rather mainly related to the addition of nutrients and organic carbon to the soil (Mayer et al. 2010). Indeed, it is difficult to separate the effects of the Bokashi organic substrate and beneficial microorganisms in the inoculant for Effective Microorganisms treatment on plant performance (Megali et al. 2014). Mayer et al. (2010) demonstrated that the mineral and carbon resources in Bokashi alone are sufficient to increase crop yield, while beneficial microorganism application by itself had no measurable impact on yield for crop species in their investigations. However, following the same logic, the species that showed a greater response to Effective Microorganisms + Bokashi treatment than to conventional fertiliser (peas, tomatoes and spinach) suggest that the beneficial microorganisms in the Effective Microorganisms inoculant can play a role in improving crop performance, as application of conventional fertiliser should have provided more than sufficient mineral resources for optimal plant performance.

\subsection{Effect of Effective Microorganisms on yield and biodiversity in an agroecosystem}

After 70 days of growth, there was no significant treatment effect for most measured corn plant traits (for chlorophyll levels $F_{1,5}=1.12, p=0.31$; for height of the plants $F_{1,5}=$ $0.96, p=0.37$; for seed dry weight $F_{1,5}=2.49, p=0.18$; for plant dry biomass $F_{1,5}=3.23, p=0.13$ ). Nevertheless, Effective Microorganisms treatment corresponded to a significant $7.3 \%$ increase in water content in corn plants $\left(F_{1,10}=2.74, p=\right.$ $0.03)$.

Enhanced water uptake alone may be of interest to horticulturalists and ecologists working in changing environments (Brouder and Volenec 2008). In addition to the implications for nutrient uptake in bulk flow, especially when soil nutrients may be relatively scarce, increasing water content holds other considerations. Shifts to precipitation regimes associated with climate change (Karl and Trenberth 2003) and ever-increasing salinization of irrigated lands (Yeo 1999) are challenges that will only become more difficult in the coming century for land managers protecting crops or any terrestrial system. However, as with growth and yield, Effective Microorganisms' effects on water relations may be species-specific or dependent on soil type and merit localised testing before the soil inoculant can be employed to battle the negative effects of drought and soil salinity with any certainty of benefits.

Additional effects of beneficial microorganism treatment in the field resulted in nuanced shifts in insect diversity. Overall, squares with Effective Microorganisms were visited by 222 species, including 40 herbivores, 66 predators and 116 other species, whereas control squares, without Effective Microorganisms, were visited by 194 insect species including 33 herbivores, 53 predators and 108 other species. The Mantel test indicated that Effective Microorganisms had no effect on overall insect diversity $(p=0.10)$. Permutation ANOVAs of Shannon Indices for the functional groups "herbivores" and non-herbivorous/non-predatorial insects, categorised as "other", also indicate no significant difference between Effective Microorganisms and controls. However, predatory insects (including spiders, predatory bugs, parasitoid wasps and flies) experienced a strongly significant $26.0 \%$ decrease in diversity 
Table 1 Volatile organic compounds emission on corn plants

\begin{tabular}{lllllr}
\hline No. & Comp. name & no_EM/no_herb & EM/no_herb & EM/herb & no_EM/herb \\
\hline 1 & un. monoterpene & $1.01 \pm 0.1$ & $1.07 \pm 0.22$ & $1.1 \pm 0.26$ & $1.26 \pm 0.25$ \\
2 & un. monoterpene & $0.27 \pm 0.02$ & $0.37 \pm 0.1$ & $0.4 \pm 0.08$ & $0.38 \pm 0.07$ \\
3 & un. & $0.33 \pm 0.07$ & $0.24 \pm 0.05$ & $0.55 \pm 0.3$ & $0.96 \pm 0.09$ \\
4 & un. monoterpene & $0.27 \pm 0.04$ & $0.24 \pm 0.05$ & $0.26 \pm 0.06$ & $0.29 \pm 0.05$ \\
5 & un. monoterpene & $0.13 \pm 0.04$ & $0.06 \pm 0.03$ & $0.33 \pm 0.23$ & $0.31 \pm 0.08$ \\
6 & un. monoterpene & $0.77 \pm 0.09$ & $0.75 \pm 0.14$ & $0.73 \pm 0.14$ & $0.87 \pm 0.14$ \\
7 & Linalool & $0.8 \pm 0.28$ & $0.43 \pm 0.11$ & $1.92 \pm 1.37$ & $5.62 \pm 0.93$ \\
8 & un. & $9.92 \pm 3.72$ & $2.91 \pm 0.92$ & $3.75 \pm 0.84$ & $27.13 \pm 5.07$ \\
9 & un. monoterpene & $0.47 \pm 0.05$ & $0.59 \pm 0.22$ & $0.71 \pm 0.24$ & $0.8 \pm 0.22$ \\
10 & un. monoterpene & $1.08 \pm 0.13$ & $1.16 \pm 0.23$ & $1.23 \pm 0.19$ & $1.25 \pm 0.24$ \\
11 & un. monoterpene & $0.01 \pm 0.01$ & $0.03 \pm 0.02$ & $0.01 \pm 0.01$ & $0.1 \pm 0.01$ \\
12 & Cyclosativene & $3.82 \pm 0.6$ & $3.86 \pm 0.76$ & $2.85 \pm 0.77$ & $5.18 \pm 0.19$ \\
13 & (E)- $\beta$-caryophyllene & $4.76 \pm 1.45$ & $2.48 \pm 0.36$ & $4.45 \pm 3.45$ & $21.05 \pm 5.22$ \\
14 & Trans-bergamotene & $0.36 \pm 0.06$ & $0.37 \pm 0.04$ & $0.29 \pm 0.09$ & $0.51 \pm 0.19$ \\
15 & $\beta$-farnesene & $1.1 \pm 0.59$ & $0.18 \pm 0.04$ & $3.43 \pm 3.28$ & $29.19 \pm 12.08$ \\
16 & un. sesquiterpene & $0.48 \pm 0.14$ & $0.36 \pm 0.04$ & $0.52 \pm 0.33$ & $2.31 \pm 0.72$ \\
17 & un. sesquiterpene & $0.16 \pm 0.01$ & $0.21 \pm 0.07$ & $0.16 \pm 0.07$ & $0.24 \pm 0.1$ \\
18 & un. sesquiterpene & $1.12 \pm 0.11$ & $1.21 \pm 0.15$ & $1.07 \pm 0.24$ & $2.08 \pm 0.23$ \\
19 & un. sesquiterpene & $0.48 \pm 0.18$ & $0.58 \pm 0.16$ & $0.77 \pm 0.24$ & $1.47 \pm 0.54$ \\
\hline
\end{tabular}

Shown are the means \pm 1 SE of individual compounds emitted by corn plants under different Effective Microorganisms (EM) treatment and herbivore (herb) treatment regimes

un. unidentified

${ }^{\mathrm{a}}$ The compound name is given when the compound was identified by comparison with pure standards on corn grown on Effective Microorganisms-treated soil $\left(F_{1}\right.$, $10=33.17, p<0.001$ ). Below, Effective Microorganisms soil inoculation is shown to inhibit the production of herbivoreinduced volatile organic compounds in corn plants, which are known to attract predators of herbivorous insects (see Sect. 3.4 below), similar results are also seen by (Turlings and Wäckers 2004). The significance of volatile organic compounds (VOCs) to plant defence has been further explored by Kaplan and Lewis (2015) in a discussion on the addition of VOCs to crop fields as a complementary measure for biological control of herbivore pests. If beneficial microorganisms are somehow reducing corn's ability to release volatiles that attract predators of the corn's herbivorous enemies, these findings could hold serious implications for farmers operating in areas with high abundance and diversity of herbivorous insects.

\subsection{Effect of Effective Microorganisms on volatile induction}

Defence and resistance of $Z$. mayz against $S$. littoralis were negatively affected by Effective Microorganisms treatment in the greenhouse. Plant indirect defences, measured as total volatile organic compounds (Kessler and Heil 2011), were affected by both Effective Microorganisms and herbivore treatment. Overall, we identified 19 individual volatile organic compounds emitted by corn plants (either in healthy or herbivore damaged state, Table 1).

When herbivores were present for 4 days, plants augmented VOC emissions by $272 \%$ (Fig. 4a, herbivore effect; $F_{1}$, ${ }_{12}=3.48, p=0.01$ ), but this was true only for control (nonEffective Microorganisms-treated) plants (Fig. 4a), Effective a) Volatile production

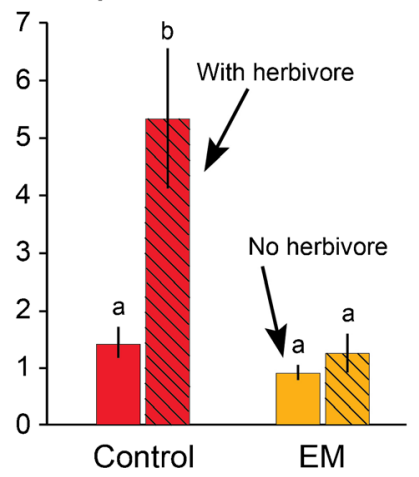

b) Caterpillar mass

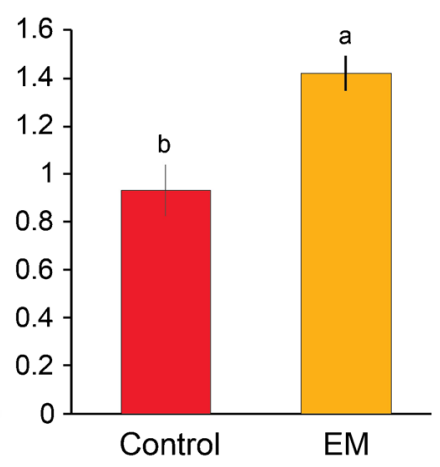

Fig. 4 Effective Microorganisms (EM) effect on plant defences and resistance. Shown is a) plant indirect defences measured as mean total volatile organic compound content (ng/h) with no herbivory (plain bars) and herbivory by Spodoptera littoralis caterpillars (hatched bars), b) mean plant resistance measured as biomass (mg dry weight) of S. littoralis larvae after 4 days of feeding on EM-treated (orange bars) or on non-treated control (red bars) corn plants. Bars are means \pm 1 SE. Letters above bars means significant differences across EM and herbivore treatments $(p<0.05$, Tukey HSD test) 
Microorganisms' effect; $F_{1,12}=4.09, p=0.01$, and Effective Microorganisms by herbivore interaction; $F_{1,12}=4.09, p=$ 0.01. Indeed, when plants were inoculated with Effective Microorganisms, no induction by herbivores of volatiles was observed. Effective Microorganisms treatment likewise significantly reduced plant resistance (measured as increased herbivore biomass) by $51.4 \%$ (Fig. $4 \mathrm{~b}, F_{1,18}=14, p=0.001$ ).

VOCs are a key adaptation in innate, inducible plant defences (Turlings and Wäckers 2004) and our results strongly suggest that Effective Microorganisms inhibit their production in corn (Fig. 4a). Further, data suggest Effective Microorganisms reduces plant resistance as seen in an increase in biomass of $S$. littoralis larvae when allowed to feed on treated and untreated corn (Fig. 4b). We found similar results with tomatoes in a separate study, in which Effective Microorganisms soil application interfered with plant signalling pathways that trigger upregulation of inducible defences after herbivore attack (Megali et al. 2014). This inhibition of innate plant defences renders Effective Microorganisms not only ineffective as a means of pest management, but could also potentially endanger efforts in safeguarding the harvest against herbivory. However, as with growth and yield, Effective Microorganisms' effects on plant defence and resistance may vary with crop species, and to a lesser extent, local soil ecology.

\section{Conclusion}

In accordance with predictions multiple axes of support were found for Effective Microorganisms being beneficial for plant growth and yield. Nevertheless, strong speciesspecific responses to inoculation demand the attention of curious farmers. In the field, Effective Microorganisms increased water content in corn plants, however, those plants were colonised at a lower abundance and diversity of predatory insect species. In accordance, Effective Microorganisms inhibits the production of volatile organic compounds for predator attraction in corn and renders the plants more palatable to caterpillar pests. Taken together, results indicate a positive effect of Effective Microorganisms on crop production but negative effects on plant defences that should be considered for future cost/benefit analyses. Implications for horticulturalists interested in exploiting alternatives to chemical fertilisers and synthetic pesticides are that localised, speciesspecific field tests should be performed before broad application of "beneficial microorganism" soil inoculants. Even if crops respond positively in terms of growth and yield, application of proven pest management techniques may be necessary to protect the harvest for market. However, multiple studies, including the experiments presented here, demonstrate that Effective Microorganisms can be an effective form of biofertiliser for several crop species, matching or even outperforming plants treated with conventional chemical fertiliser.

Acknowledgments The current project was supported by Swiss National Science Foundation Ambizione grant PZ00P3 131956/1 to SR. We are grateful to Ueli Rothenbuhler for providing Effective Microorganisms substrate and to Eros Gentilini for stimulating the ideas.

\section{References}

Adesemoye AO, Kloepper JW (2009) Plant-microbes interactions in enhanced fertilizer-use efficiency. Appl Microbiol Biotechnol 85(1):112. doi:10.1007/s00253-009-2196-0

Altieri MA (1995) Agroecology: the science of sustainable agriculture. Westview Press, Boulder

Bashan Y (1998) Inoculants of plant growth-promoting bacteria for use in agriculture. Biotechnol Adv 16(4):729-770. doi:10.1016/s07349750(98)00003-2

Brouder SM, Volenec JJ (2008) Impact of climate change on crop nutrient and water use efficiencies. Physiol Plant 133(4):705-724. doi:10. 1111/j.1399-3054.2008.01136.x

Compant S, Duffy B, Nowak J, Clément C, Barka EA (2005) Use of plant growth-promoting bacteria for biocontrol of plant diseases: principles, mechanisms of action, and future prospects. Appl Environ Microbiol 71(9):4951-4959. doi:10.1128/aem.71.9.4951-4959. 2005

Cóndor Golec AF, Lokare C, González Pérez P (2007) Effective Microorganisms: myth or reality? Rev Peru Biol 14(2):315-319

Foley JA, Ramankutty N, Brauman KA, Cassidy ES, Gerber JS, Johnston M, Mueller ND, O'Connell C, Ray DK, West PC, Balzer C, Bennett EM, Carpenter SR, Hill J, Monfreda C, Polasky S, Rockstrom J, Sheehan J, Siebert S, Tilman D, Zaks DPM (2011) Solutions for a cultivated planet. Nature 478(7369):337-342. doi:10.1038/ nature 10452

Gevao B, Semple KT, Jones KC (2000) Bound pesticide residues in soils: a review. Environ Pollut 108(1):3-14. doi:10.1016/s0269-7491(99) 00197-9

Green RE, Cornell SJ, Scharlemann JPW, Balmford A (2005) Farming and the fate of wild nature. Science 307(5709):550-555. doi:10. 1126/science. 1106049

Hayat R, Ali S, Amara U, Khalid R, Ahmed I (2010) Soil beneficial bacteria and their role in plant growth promotion: a review. Ann Microbiol 60(4):579-598. doi:10.1007/s13213-010-0117-1

Higa T (2000) What is EM technology? EM World J 1:1-6

Javaid A (2010) Beneficial microorganisms for sustainable agriculture. In: Lichtfouse E (ed) Genetic engineering, biofertilisation, soil quality and organic farming, vol 4. Sustainable agriculture reviews. Springer, Netherlands, pp 347-369. doi:10.1007/978-90-4818741-6 12

Johansson JF, Paul LR, Finlay RD (2004) Microbial interactions in the mycorrhizosphere and their significance for sustainable agriculture. FEMS Microbiol Ecol 48(1):1-13. doi:10.1016/j.femsec.2003.11. 012

Kaplan I, Lewis D (2015) What happens when crops are turned on? Simulating constitutive volatiles for tritrophic pest suppression across an agricultural landscape. Pest Manag Sci 71(1):139-150. doi: $10.1002 / p s .3779$

Karban R, Baldwin IT (1997) Induced responses to herbivory. The University of Chicago Press, Chicago

Karl TR, Trenberth KE (2003) Modern global climate change. Science 302(5651):1719-1723. doi:10.1126/science.1090228 
Kessler A, Heil M (2011) The multiple faces of indirect defences and their agents of natural selection. Funct Ecol 25(2):348-357. doi:10.1111/ j. 1365-2435.2010.01818.x

Kong L (2014) Maize residues, soil quality, and wheat growth in China. A review. Agron Sustain Dev 34(2):405-416. doi:10.1007/s13593013-0182-5

Lentendu G, Wubet T, Chatzinotas A, Wilhelm C, Buscot F, Schlegel M (2014) Effects of long-term differential fertilization on eukaryotic microbial communities in an arable soil: a multiple barcoding approach. Mol Ecol 23(13):3341-3355. doi:10.1111/mec.12819

Mayer J, Scheid S, Widmer F, Fließbach A, Oberholzer H-R (2010) How effective are 'Effective Microorganisms ${ }^{\circledR}$ (EM)'? Results from a field study in temperate climate. Appl Soil Ecol 46(2):230-239. doi:10.1016/j.apsoil.2010.08.007

Megali L, Glauser G, Rasmann S (2014) Fertilization with beneficial microorganisms decreases tomato defenses against insect pests. Agron Sustain Dev 34(3):649-656. doi:10.1007/s13593-0130187-0

Oksanen J, Blanchet FG, Kindt R, Legendre P, Minchin PR, O'Hara RB, Simpson GL, Solymos P, Stevens MHH, Wagner H (2013) Vegan: community ecology package. 2.0-10 edn http://vegan.r-forge.rproject.org/

Pineda A, Zheng S-J, van Loon JJA, Pieterse CMJ, Dicke M (2010) Helping plants to deal with insects: the role of beneficial soil- borne microbes. Trends Plant Sci 15(9):507-514. doi:10.1016/j. tplants.2010.05.007

Stoate C, Boatman ND, Borralho RJ, Carvalho CR, de Snoo GR, Eden P (2001) Ecological impacts of arable intensification in Europe. J Environ Manag 63(4):337-365. doi:10.1006/jema.2001.0473

Team RDC (2011) Vienna, Austria: R foundation for statistical computing

Tilman D, Cassman KG, Matson PA, Naylor R, Polasky S (2002) Agricultural sustainability and intensive production practices. Nature 418(6898):671-677. doi:10.1038/nature01014

Turlings TCJ, Wäckers F (2004) Recruitment of predators and parasitoids by herbivore-injured plants. In: Cardé RT, Millar JG (eds) Advances in insect chemical ecology. Cambridge University Press

van de Voorde TFJ, van der Putten WH, Bezemer TM (2012) Soil inoculation method determines the strength of plant-soil interactions. Soil Biol Biochem 55:1-6. doi:10.1016/j.soilbio.2012.05.020

Vitousek PM, Mooney HA, Lubchenco J, Melillo JM (1997) Human domination of earth's ecosystems. Science 277(5325):494-499. doi:10.1126/science.277.5325.494

Wheeler RE (2010) multResp() lmPerm. The R project for statistical computing http://www.r-project.org/

Yeo A (1999) Predicting the interaction between the effects of salinity and climate change on crop plants. Sci Hortic 78(1-4):159-174 